\title{
PORTRAIT DES CONSÉQUENCES ASSOCIÉES AUX MALTRAITANCES INFANTILES INTRAFAMILIALES À PARTIR D'UNE RECENSION D'ÉCRITS SCIENTIFIQUES
}

Iliona WATTEL ${ }^{1 *}$

'École de psychologie, Université Laval, Québec, QC, Canada

*lliona.wattel.1@ulaval.ca

\section{Pour citer l'article}

Wattel, I. (2020). Portrait des conséquences associées aux maltraitances infantiles intrafamiliales à partir d'une recension d'écrits scientifiques. Psycause: Revue scientifique étudiante de l'École de psychologie de l'Université Laval, 10(1), 28-44.

\section{Droits d'auteur}

(C) 2020 Wattel. Cet article est distribué en libre accès selon les termes d'une licence Creative Commons Attribution 4.0 International (de type CC-BY 4.0) qui permet l'utilisation du contenu des articles publiés de façon libre, tant que chaque auteur ou autrice du document original à la publication de l'article soit cité(e) et référencé(e) de façon appropriée. 


\title{
PORTRAIT DES CONSÉQUENCES ASSOCIÉES AUX MALTRAITANCES INFANTILES INTRAFAMILIALES À PARTIR D’UNE RECENSION D'ÉCRITS SCIENTIFIQUES
}

\author{
Iliona WATTEL ${ }^{1 *}$ \\ 'École de psychologie, Université Laval, Québec, QC, Canada \\ *lliona.wattel.1@ulaval.ca
}

\begin{abstract}
Résumé
Les maltraitances infantiles intrafamiliales, par leur prévalence élevée au sein de notre société, constituent un problème majeur de santé publique. Toutefois, elles restent encore trop souvent tues, voire niées, alors que leurs conséquences sont pourtant bien présentes. Cet article a pour but de sensibiliser à l'importance de ces conséquences à travers une recension des écrits à ce sujet. Dans un premier temps, les maltraitances physiques, psychologiques et sexuelles, ainsi que la négligence seront définies et leur impact au sein de la famille sera expliqué. Les conséquences du trauma seront abordées, entre autres quant aux manifestations infantiles, aux comportements à risque et aux troubles psychologiques chez l'adolescent. Enfin, la recension portera aussi sur l'impact des maltraitances infantiles dans les différentes sphères de la vie de l'adulte, ainsi que sur la résilience et la survivance des symptômes liés aux maltraitances.
\end{abstract}

\section{Mots-clés : Maltraitances infantiles, Dysfonctionnements familiaux, Inceste, Violences}

\section{Abstract}

\begin{abstract}
Due to their high prevalence in our society, intra-familial child abuse is a major public health problem. However, they are still too often kept silent or denied, even though their consequences are very much present. The aim of this article is to raise awareness of the importance of these consequences through a review of the literature on the subject. First, physical, psychological and sexual abuse, as well as neglect, will be defined and their impact within the family will be explained. The consequences of the trauma will be discussed, among others, with regard to childhood manifestations, risk behaviours and psychological disorders in adolescents. Finally, the review will also examine the impact of child maltreatment in different spheres of adult life, as well as resilience and survival of symptoms related to maltreatment.
\end{abstract}

\section{Keywords : Child abuse, Family dysfunctions, Incest, Violence}

La maltraitance infantile est définie par l'Organisation Mondiale de la Santé comme «toute forme de violences, d'atteintes ou de brutalités physiques et mentales, d'abandon et de négligence, de mauvais traitements ou d'exploitation, y compris la violence sexuelle» (Krug, Dahlberg, Mercy, Ziwi, \& LozanoAscencio, 2002, p.59). Bien qu'elle puisse avoir lieu dans plusieurs contextes, comme à l'école par exemple, c'est dans le cercle familial qu'elle est la plus courante. Ces sévices sont longtemps restés tabous dans la société, voire ritualisés dans certaines cultures (Lévy, 2008). Ce n'est qu'au XXème siècle qu'apparait l'idée de protéger les enfants, notamment avec la Déclaration des Droits de l'Enfant, rédigée en 1959. La reconnaissance des sévices infantiles s'est faite progressivement en milieu médical, mais il a fallu attendre 2006 pour que l'Organisation Mondiale de la Santé les catégorise comme un problème de santé publique majeur et souligne la nécessité d'investir pour leur prévention (Benarous, Consoli, Raffi, \& Cohen, 2014).

Pourtant, dans nos sociétés occidentales, la maltraitance infantile est encore largement répandue et très souvent passée sous silence (Krug \& coll., 2002). Ces violences peuvent prendre de multiples formes et entraîner de lourdes conséquences pour le sujet qui en est victime, et ce tout au long de sa vie. L'objectif de cet article est d'exposer les raisons pour lesquelles ces maltraitances constituent un problème majeur de santé publique et pourquoi leurs conséquences sont si importantes pour les victimes. Cette recension définira, dans un premier temps, l'étiologie des différents types de sévices infantiles au sein de la famille. II 
s'agira ensuite d'investiguer la façon dont le sujet se construit en réponse à ces violences tout au long de sa vie, à travers le processus de résilience et la survivance des symptômes du traumatisme.

Avant d'aborder les violences infantiles intrafamiliales, il faut tout d'abord définir le concept de la famille ainsi que son fonctionnement.

\section{La famille: rôles, fonctionnement et dysfonctionnements}

\section{Les rôles et le fonctionnement normal}

Selon Lévi-Strauss (1986), une famille est une communauté d'individus réunis par des liens de parenté et d'alliance existant dans toutes les sociétés humaines. Au-delà de ces liens biologiques et culturels, la famille est avant tout une structure conditionnant le développement de l'enfant en lui apportant les bases de l'éducation propre à une culture, la sécurité et les denrées vitales. Elle est donc censée subvenir aux besoins de l'enfant tout en lui apportant amour et protection, indispensables à son développement. Son but est de lui donner un sentiment d'identité et d'appartenance, par la filiation et la place au sein des différentes générations. La famille est régie par les interdits primaires de l'inceste et du meurtre, qui structurent également l'ensemble des société humaines.

Il est donc nécessaire de s'interroger sur l'exercice de ces droits et de ces devoirs, et de déterminer le fonctionnement normal d'une famille avant d'aborder ses dysfonctionnements.

L'éducation faite par les parents permet à l'enfant de s'adapter à la société dans laquelle il évolue et de nouer des liens sociaux. Les parents jouent un rôle essentiel auprès de l'enfant, car ils représentent une figure primordiale d'attachement qui remplit une fonction contenante et structurante. L'autorité parentale occupe un rôle central dans l'éducation. L'article 371-1 du Code Civil français (2019) la définit comme «un ensemble de droits et de devoirs ayant pour finalité l'intérêt de l'enfant. Elle appartient aux pères et aux mères jusqu'à la majorité ou l'émancipation de l'enfant pour le protéger dans sa sécurité, sa santé et sa moralité, pour assurer son éducation et permettre son développement, dans le respect dû à sa personne». L'établissement du lien parent-enfant, dès les débuts de la relation, est donc primordial pour le déroulement futur de l'éducation, et met en jeu le climat du foyer dans lequel évolueront les membres de la famille. Le comportement parental se base, d'après Houzel, sur deux dimensions majeures (Solis-Ponton, 2002). Premièrement, l'axe permissivité/contrainte concerne l'interférence des parents dans la vie des enfants, les limites qu'ils établissent, la rigueur avec laquelle ils appliquent les règles et le mode d'exercice de leur pouvoir parental. Deuxièmement, l'axe chaleur/hostilité concerne l'engagement des parents dans le bienêtre de leur enfant, leurs réponses à ses besoins, le temps consacré à ses activités, leur sensibilité face à ses états émotionnels. De ces dimensions découlent une multitude de stratégies éducatives et de styles parentaux, qui contribueront à façonner le développement de l'enfant.

Selon Widmer, Kellerhals et Lévy (2004), la structure de la famille détermine les relations et les interactions entre ses membres et s'analyse selon trois dimensions. La cohésion interne, tout d'abord, désigne la manière dont les membres de la famille se relient au groupe. L'intégration externe, ensuite, concerne la façon dont le groupe s'ouvre à l'extérieur. La régulation, quant à elle, se traduit par la coopération des membres dans le groupe. Les interactions comportementales, affectives et fantasmatiques entre le nourrisson et ses parents vont être déterminantes pour leur relation future (Delion, 2011). Les interactions comportementales sont directement observables et consistent en un contrepoint du comportement du parent chez le bébé. En effet, un nourrisson qui grandit dans un environnement serein et sécuritaire connaîtra un développement optimal, contrairement à la progéniture d'un parent dépressif, par exemple (Cohn \& Tronick, 1989). Les interactions affectives, ensuite, définissent l'ensemble des vécus émotionnels et affectifs entre le parent et le bébé. Ceux-ci sont rendus possibles grâce aux compétences précoces du nourrisson, qui instaurent un processus d'attachement et de recherche vis-à-vis du parent. On peut assimiler ce concept d'accordage affectif (ou harmonie), décrit par Stern (Ciccone, Gauthier, Glose, \& Stern, 2008), à la théorie de l'attachement (ou bonding) de Bowlby (Dugravier \& Barbey-Mintz, 2015), qui décrit la nécessité du bébé de s'attacher à celui qui répond à ses besoins primaires. Cette première relation amorcera la qualité des interactions ultérieures dans la dyade parent- 
enfant, et déterminera le sentiment de sécurité ou d'insécurité de l'enfant. Les interactions fantasmatiques, enfin, relatent l'ensemble des représentations conscientes et inconscientes du parent au sujet de son identité et de celle de son enfant.

Les styles familiaux sont nombreux et complexes, et le fonctionnement du groupe familial est bien loin de se limiter au modèle de la «bonne famille », mythe inhérent à notre société, qui considère que les membres de celle-ci doivent fonctionner de manière harmonieuse et uniforme. Il est effectivement possible que cette structure, censée contribuer au développement et au bien-être de l'enfant, se révèle être néfaste voire destructrice pour lui

\section{Les dysfonctionnements familiaux}

Afin d'aborder les différents aspects des maltraitances infantiles intrafamiliales ainsi que leurs conséquences, I'ouvrage de Gruyer, Sabourin et Fadier-Nisse (1991) sur ce sujet a été choisi comme référence principale en raison de sa pertinence et de sa complétude. II faut toutefois souligner que, malgré ces qualités, il s'agit d'une source plutôt ancienne.

Les maltraitances infantiles peuvent être le résultat d'un mauvais fonctionnement des interactions et des axes de parentalité décrits plus hauts. Cela peut être directement lié à l'un des parents, au couple parental, ou encore au dysfonctionnement de la structure familiale.

\section{L'addiction des parents: un objet de dépendance extérieur.}

Dans le cas des parents alcooliques ou toxicomanes, la dépendance peut d'abord affecter les premières interactions entre la mère et le bébé, et ainsi entraîner un désinvestissement de la part de celle-ci à l'égard du nourrisson. Cela provient du fait que celui-ci est dépendant de sa mère, alors qu'elle est elle-même dépendante de l'objet addictif, qui interfère alors dans sa relation avec l'enfant (Delion, 2011). De plus, la culpabilité fréquemment ressentie par les parents toxicomanes au sujet de leurs difficultés à remplir leur rôle auprès de l'enfant rend la prise en charge difficile, et multiplie les facteurs de vulnérabilité pour ce dernier (Rosenbulm, 2000).

\section{La violence conjugale: I'enfant en tant que victime indirecte.}

Une mauvaise relation entre les parents se répercute sur l'ensemble du fonctionnement de la famille, et donc également sur l'enfant. Dans le cas de violences conjugales celui-ci devient la victime indirecte de ces actes car il en est témoin et se retrouve dans une situation impossible à assimiler. L'enfant assiste aux scènes de violence dans l'impuissance et la passivité, ce qui provoquera une angoisse pouvant s'associer à un traumatisme (Razon \& Metz, 2011).

\section{Le dysfonctionnement de l'ensemble de la sphère familiale: autorité abusive et inceste.}

Parmi les structures familiales biaisées, on retrouve les dictatures familiales et les familles incestueuses. Dans les dictatures familiales, tout d'abord, I'abus d'autorité et la violence sont banalisés. Les membres de la famille sont soumis à la tyrannie d'un des parents, qui instaure des règles très strictes et immuables. Pour ce "chef de famille», il est question de déshumaniser ses "sujets», de les réduire à l'état de possessions soumises à son bon vouloir. Le respect et l'ordre sont alors les maîtres mots, et toute incartade est perçue comme une atteinte au «bon fonctionnement» de la famille (Gruyer \& coll., 1991).

Dans les familles incestueuses, ensuite, I'interdit primaire de l'inceste ne vient ni réguler ni limiter les relations. C'est l'effraction dans le corps et la sphère privée qui est la norme. On assiste à une absence de différenciation entre les membres: les rôles, les générations et les âges sont confus, les relations ambiguës. Les limites physiques et affectives sont brouillées et inadéquates, la notion d'appartenance est inexistante. Cette absence de frontière entre les corps se retrouve dans la structure même de la famille. Ses membres s'organisent autour de cette charpente biaisée, et il est impossible pour l'enfant $d^{\prime} y$ établir des repères pour se construire des bases saines.

Dans ces familles règne un climat de danger constant, alimenté par l'emprise et la manipulation. La loi du silence bâillonne symboliquement les victimes du parent maltraitant sous le sceau de la terreur et la loyauté, ce qui peut les mener à douter de leurs perceptions et de la réalité. Les menaces, claires ou implicites, les empêchent d'avoir recours à une 
aide extérieure et maintient les sévices subis dans le secret. L'homéostasie est inhérente à ces structures familiales: le changement est perçu comme une erreur, tout doit rester figé dans ce climat malsain et insécure (Rouyer \& Drouet, 1994). Les maltraitances intrafamiliales peuvent mener à la négation de l'identité de l'enfant, de son droit de vivre et d'être une personne à part entière: il n'est alors plus que l'objet de son agresseur, objet de possession, de désir et de servitude.

La famille peut donc s'avérer néfaste voire destructrice pour l'enfant, et plusieurs types de maltraitances peuvent découler des dysfonctionnements familiaux.

\section{Les différents types de maltraitances}

\section{Les violences physiques}

Les violences physiques sont les plus visibles, car elles laissent généralement des traces sur le corps de l'enfant, mais demeurent encore largement ignorées voire niées par l'entourage de ce dernier (Greco, 2015). Elles se traduisent par des coups et blessures perpétrés par le parent, résultant d'une discipline biaisée et d'une répression excessive. Ces sévices constituent un abus de pouvoir de la part du parent, alors que sa fonction première est d'établir une discipline claire et une bonne communication avec l'enfant (Montandon \& Kellerhals, 1991). Dans les cas les plus extrêmes, ces violences peuvent conduire à l'infanticide.

\section{L'abus de maternage et l'inceste}

Les sévices sexuels infantiles font partie des violences physiques, mais relèvent $d^{\prime} u n$ tout autre type de maltraitance.

L'Institut de Victimologie (2019) les définit comme suit:

Une activité sexuelle à laquelle une victime est incitée ou contrainte de participer par un agresseur sur lui-même, sur elle-même ou sur une tierce personne; contre son gré, ou par manipulation affective, physique, matérielle ou usage d'autorité, de manière évidente ou non; que l'agresseur soit connu ou non, qu'il y ait ou non évidence de lésions ou de traumatisme physique ou émotionnel, et quel que soit le sexe des personnes impliquées.
Ces abus sont répartis en trois catégories: les agressions sans contact physique, les agressions avec contact physique mais sans pénétration, et les agressions avec pénétration.

On parle d'abus sexuel intrafamilial lorsque l'agresseur est un membre de la famille immédiate (père, mère, conjoint(e) de ceux-ci, membre de la fratrie). Cet article portera plus spécifiquement sur l'inceste, qui se définit selon le Centre National de Ressources Textuelles et Lexicales (CNRTL) par des relations sexuelles prohibées entre des personnes de la même famille. Il est question ici de l'inceste entre un enfant et son parent, qui est le fruit d'une séduction narcissique de ce dernier sur sa progéniture, d'un détournement de sa fonction et d'une transgression fondamentale des interdits structurant la famille (Gruyer \& coll., 1991).

L'abus de maternage, ou nursing pathologique, fait également partie des agressions sexuelles et se caractérise par des abus dans les soins de l'enfant, des toilettes invasives faisant intrusion sur son corps et des comportements de séduction. Ces conduites découlent du sentiment de propriété de l'adulte maternant sur sa progéniture et du besoin de fusionner avec son bébé, ce qui crée dès le départ une relation pathologique avec celui-ci se traduisant par des premières interactions inadéquates.

\section{La maltraitance psychologique}

Ce type de violence est très subtile, car il laisse des traces invisibles, mais tout aussi destructrices dans le psychisme de l'enfant. II est synonyme d'harcèlement moral, qui se définit comme «des propos ou comportements répétés ayant pour objet ou pour effet une dégradation des conditions de vie de la victime, se traduisant par une altération de sa santé physique ou mentale » dans l'article 222-33-2-2 du Code Pénal français (2018). II s'agit en fait de blesser avec des mots, d'humilier, de menacer et de rabaisser.

La violence psychologique accompagne nécessairement les deux autres types de maltraitance cités plus haut, mais peut également se manifester seule. Généralement, elle se traduit par des sous-entendus, des messages subtiles, des attaques répétées et constantes qui conditionnent le développement de l'enfant, renforçant alors l'emprise de son agresseur par son infériorité et son incapacité à se défendre. 
La dyade parent-enfant ne se base ainsi plus sur des axes normaux, mais sur un rapport de domination qui instaure un mode de communication biaisé au sein du groupe familial.

\section{La négligence}

Cette dernière forme de maltraitance se définit comme une absence ou une insuffisance de soins, de protection et de surveillance. II s'agit dans ce cas d'un désinvestissement du parent à l'égard de son enfant: il ne remplit pas sa fonction primaire, qui est de répondre aux besoins fondamentaux de sa progéniture. Cela entraîne, en plus de graves carences affectives, des conséquences telles qu'un retard de développement chez les enfants en bas âge, voire à leur mort par sous-alimentation, accident ou infection.

Les conséquences de ces maltraitances affecteront le sujet de façon différente selon les étapes de son développement.

\section{Les conséquences des maltraitances chez l'enfant}

\section{Le traumatisme}

Avant de parler des symptômes à court-terme qu'entraînent ces violences, il faut d'abord définir ce qu'est un traumatisme, de quelle façon il se manifeste et quels mécanismes sont impliqués dans son développement.

Thinès (1975) définit le traumatisme comme «Tout événement subi, brutal, entraînant pour le sujet qui en est victime des transformations plus ou moins profondes, plus ou moins réversibles. » Les manifestations du traumatisme se font en plusieurs temps, et les symptômes qui en découlent diffèrent selon la personne qui le subit.

\section{Les manifestations immédiates.}

Le temps de l'effraction traumatique, tout d'abord, donne lieu à un état de sidération. II s'agit d'une brève éclipse, un vide total de la pensée qui rend le sujet incapable d'élaborer et d'agir. Celui-ci se trouve en effet confronté à quelque chose qui en fait pas de sens, et auquel il ne peut faire face. Cet état peut mener à une dissociation entre le corps et le mental; c'est-à-dire une impression de sortir de son corps, de «ne plus être là » (Lebigot, 2016).

L'effroi laisse ensuite place au «stress», que l'on peut décrire comme un "syndrome d'adaptation général» (Selye, 1936), une "réaction aigüe à un facteur de stress» ou un «stress adapté» (Guillet, 2012). ॥ s'agit de la réaction, proprement dite, à l'événement traumatique. Elle se traduit par un état d'alerte et de mobilisation cognitive, affective, volitionnelle et comportementale ayant pour but d'élaborer une décision et d'adopter les gestes adéquats pour faire face à la menace.

\section{Les manifestations post-immédiates.}

La période post-immédiate survient après un temps de latence plus ou moins long selon les sujets et peut se caractériser par l'apparition de reviviscences, d'états dépressifs, de troubles du sommeil ou encore d'une anxiété phobique traduisant un sentiment d'insécurité constant. Ces symptômes risquent, alors, d'évoluer vers un syndrome de stress post-traumatique.

\section{Le trouble de stress post-traumatique.}

Le trouble de stress post-traumatique (TSPT), également appelé "névrose traumatique», relève d'un trouble sévère dont les symptômes se révèlent ou se prolongent dans le temps, au-delà du moment du traumatisme. Plusieurs facteurs peuvent favoriser son développement chez l'individu, tels que le vécu antérieur, l'organisation structurelle de la personnalité, ou encore la maîtrise des affects et les ressources de résilience (Scharbach, 2018).

Plus le traumatisme intervient tôt dans le développement de l'enfant, plus sa portée sera conséquente. Chez le jeune sujet, la fragilité n'est pas forcément plus importante que chez l'adulte, mais l'impact du traumatisme est différent. II est plus difficile pour lui, par exemple, de comprendre et d'expliquer ce qu'il a vécu, car il n'a pas forcément les mots pour se le représenter (Marcelli, 2014). Le traumatisme infantile, causé par la maltraitance, peut engendrer divers troubles développementaux. 


\section{Les symptômes différés et chroniques chez l'enfant}

Les symptômes des maltraitances infantiles affectent l'enfant sur plusieurs plans. On peut observer, tout d'abord, une hyperactivité neurovégétative dans divers domaines.

\section{Les troubles alimentaires et les douleurs somatiques.}

Au niveau alimentaire, l'enfant peut présenter un comportement anorexique ou boulimique ainsi qu'un dégoût vis-à-vis des aliments, des vomissements et des difficultés de déglutition (surtout présentes chez le nourrisson). Ces symptômes peuvent s'accompagner, en plus de troubles digestifs, d'énurésie ou d'encoprésie, qui relèvent d'une forme de régression affective et instinctuelle chez l'enfant (Gruyer \& coll., 1991).

La somatisation est également très présente chez l'enfant maltraité, et peut se manifester, par exemple, par des maux de ventre ou une fatigue extrême. Il s'agit là d'un moyen pour le corps de s'exprimer, lorsqu'il est impossible pour l'enfant de mettre des mots sur ce qu'il subit (Gruyer \& coll., 1991).

\section{Les troubles du sommeil et leur impact sur le fonctionnement cognitif.}

Sur le plan du sommeil, l'enfant peut présenter des difficultés d'endormissement, des cauchemars à répétition, des terreurs et des réveils nocturnes, une peur du coucher et du sommeil. L'incidence de ces symptômes vient renforcer les troubles que l'enfant peut présenter durant la journée; tels des difficultés d'attention et de mémoire, un manque d'imagination et d'élaboration ainsi qu'une distraction très présente, qui peuvent impacter de façon négative sa vie quotidienne (Crocq, 2014).

\section{Les troubles comportementaux et affectifs.}

L'état général de l'enfant peut être très variable d'un sujet à l'autre. On peut, par exemple, remarquer une irritabilité importante et un état de vigilance permanent chez certains, ou un retrait et un émoussement général des réactions chez d'autres.

Certaines manifestations sont caractéristiques des formes de violences spécifiques. Chez les enfants victimes d'abus sexuels, par exemple, on peut observer des comportements hypersexualisés, une recherche compulsive de contact physique ou encore un dégoût pour ce dernier (Becker, 2015). Les abus peuvent également entraîner des blocages chez l'enfant, comme par exemple un refus de se déshabiller, et des comportements d'évitement vis-à-vis son agresseur. On peut aussi observer des rituels de nettoyage excessif, qui traduisent une volonté de se laver de la souillure provoquée par l'abus, ou encore, dans les cas les plus extrêmes, une perte de la notion d'hygiène, qui révèle quant à elle une tentative de se protéger en faisant de son corps un objet de répulsion.

Concernant les violences physiques, on peut observer un repli sur soi et des gestes de retrait de la part de l'enfant. La crainte des représailles peut être si forte que celui-ci, en présence de son agresseur, n'ose plus faire un geste (Felscherinow, 1981, p.24): «Je commence à trembler dès qu'on se met à table, raconte Christiane Felscherinow dans son autobiographie, si je fais une saleté c'est un drame, si je renverse quelque chose gare à mes fesses. C'est à peine si j'ose toucher à mon verre de lait. J'ai si peur qu'il m'arrive malheur à chaque repas, ou presque.» Ces abus de pouvoir sur l'enfant peuvent entraîner une inhibition de sa capacité de révolte, ou au contraire une violence accrue envers autrui et un refus d'autorité (Gruyer \& coll., 1991).

Au cours de son développement, l'enfant victime de maltraitances intrafamiliales perd la confiance fondamentale qu'il accorde à l'adulte. Le fait que le parent maltraitant, au lieu de remplir son rôle protecteur, donne au contraire une réponse violente au besoin de l'enfant, bouleverse la vision de celui-ci vis-à-vis des rapports affectifs et familiaux, et plus largement des rapports sociaux par la suite.

Paradoxalement, l'enfant présente également une très forte angoisse de séparation envers le parent maltraitant, et se construit une image idéalisée de ce dernier dont il ne peut se défaire. II s'agit là d'une autre manifestation du TSPT infantile: les carences affectives subies par l'enfant donnent lieu à un attachement pathologique au parent et à la famille, ce qui rend impossible tout éloignement. Malgré les violences qu'il subit au sein de son foyer, l'enfant désirera y retourner et manifestera une grande souffrance si on l'en sépare. Afin de survivre psychologiquement, l'enfant s'appuie donc sur l'image hyper-idéalisée de ses 
parents pour grandir, et subira le paradoxe constant de la représentation magnifiée de son agresseur qui se heurte à la figure rigide du persécuteur (Greco, 2015). Felscherinow (1981, p.25) en témoigne: "Malgré tout, j'aime et je respecte mon père. Je le trouve de loin supérieur aux autres. J'ai peur de lui, mais son comportement me parait somme toute normal.»

\section{Les conséquences à long-terme}

\section{Un apprentissage biaisé de la vie.}

Un enfant qui a grandi dans un environnement violent se construira via un apprentissage biaisé de la vie, ce qui impactera fortement sa vision du monde et ses relations futures. Dans un milieu familial dysfonctionnel, l'enfant est exposé à des événements inadaptés à son âge, ce qui peut entraîner cette pseudomaturité caractéristique du sujet que l'on a arraché au monde de l'enfance. L'ouvrage autobiographique de Nathalie Schweighoffer (1990, p.112), qui raconte les tortures et les viols infligés par son père durant cinq ans, offre une illustration pertinente de ce concept: «Parce $q u$ 'on vieillit vite, dans mon cas. On pige vite. L'enfance reste mutilée quelque part, inachevée, perdue à jamais. Les salauds, ils vous tuent dans l'œuf. »

\section{L'impact sur l'estime de soi et les relations sociales.}

Les violences subies affectent la perception de soi et entraînent d'importantes failles narcissiques. Dans le cas des abus sexuels, l'image du corps souillé et dégradé perdure dans le psychisme du sujet, qui conservera alors le sentiment d'être sali et mutilé. L'inceste met en jeu la pulsion de mort, et l'enfant vit ce traumatisme comme un abandon de la part du parent. La victime est niée en tant que sujet, réduite à la passivité, tant et si bien que cela impactera son identité en construction (Gruyer \& coll., 1991).

En plus de l'estime de soi, les relations sociales de l'enfant sont également affectées. Les enfants maltraités estiment en effet avoir moins d'amis, s'adonnent moins souvent à des jeux infantiles, nourrissent moins d'ambitions et souffrent d'un manque de confiance en eux (Kim Oates, Forest, \& Peacock, 1985).

\section{Le silence et la culpabilité.}

La culpabilité et la honte sont omniprésentes chez les sujets violentés. Les phrases dépréciatives et les menaces proférées par l'adulte finissent par être intériorisées, entraînant alors un important sentiment de dévalorisation chez l'enfant. Celui-ci se sentira coupable de ne pas avoir pu se défendre face aux violences, mais aussi coupable vis-à-vis de son agresseur, qui parvient à retourner l'accusation de ses méfaits contre la victime. Dans le cas de Nathalie (Schweighoffer, 1990, p.199), par exemple, le père l'accuse de provoquer d'elle-même les abus qu'il lui fait subir, et la maintient dans le silence par la menace du suicide de sa mère si elle venait à apprendre la vérité. Elle finit alors par intérioriser cette menace, ainsi que la culpabilité de son père, idée envahissante avec laquelle elle se débat sans cesse et qui l'empêche d'avouer les crimes de celui-ci: «Parler, dire les choses, accuser, c'est m'accuser moi-même.»

L'enfant maltraité se trouve donc dans une impasse: il se voit dans l'incapacité de parler, par peur de ne pas être entendu, de subir des représailles de la part de son agresseur, mais surtout par crainte de trahir celui-ci et de briser le cercle de la famille. C'est ainsi qu'opère le mécanisme de la maltraitance: par la peur, la honte, la confusion et la loyauté, la loi du silence finit par être intériorisée par la victime, ce qui l'empêchera ainsi de dénoncer les actes qu'elle a subi.

\section{Le déni, le clivage de l'identité et l'amnésie dissociative.}

Le refoulement se définit comme un mécanisme de défense du psychisme contre l'intrusion du traumatisme. Il s'agit de la mise à distance, consciente ou non, d'une représentation inacceptable qui met en danger l'intégrité psychique du sujet (Bokanowsky, 2002). Ferenczi (2009) explique que face à l'impossibilité de fuir la source traumatique extérieure, celui-ci opère un clivage de sa personnalité afin de pouvoir survivre, détruisant alors sa partie sensible pour ne laisser subsister que la partie insensible et inatteignable de son être, d'où les comportements d'isolement et d'émoussement des réactions de l'enfant.

L'absence de remémoration de l'événement est également un mécanisme défensif pouvant évoluer vers une amnésie dissociative, c'est-à-dire l'incapacité 
de récupérer des souvenirs autobiographiques, voire une perte d'identité. Le dévoilement de ces souvenirs peut parfois être très tardif et entraîner d'importants bouleversements (Thomas-Antérion, 2012).

Après avoir abordé les symptômes des maltraitances infantiles chez l'enfant, il faut maintenant se pencher sur la façon dont ceux-ci s'expriment à l'adolescence.

\section{Les conséquences chez l'adolescent}

L'adolescence est une période pendant laquelle se produisent de grands bouleversements, notamment lors de l'apparition de la puberté. Le sujet se construit un cadre structurant doté d'interdits et d'exigences, et s'identifie à des images parentales pour se construire, ainsi que fonder son système de valeurs et de normes (Melgual, 2019). Lors de cette période cruciale, les violences subies par le sujet au sein de sa famille auront forcément un impact sur ces processus. L'adolescent, en effet, s'identifie à des figures parentales néfastes pour sa construction, et s'appuie sur des relations biaisées pour développer ses rapports avec autrui. Les liens étroits d'amour-possession et de haine-destruction entretenus avec son agresseur façonneront sa personnalité en devenir, pouvant alors donner lieu à de nombreuses difficultés psychologiques.

\section{Comportements à risque}

\section{Marginalité, délinquance et passages à l'acte violents.}

La marginalité, relevant d'une incapacité à adhérer aux normes sociales, peut être le produit d'une éducation marquée par la négligence, dans laquelle le sujet n'a pas intériorisé les règles d'interactions sociales et de vie en société. Sur le versant opposé, l'individu ayant grandi dans un cadre familial très rigide, régi par des règles très strictes, pourra développer une attitude de refus allant au-delà de la remise en cause de l'autorité apparaissant généralement à l'adolescence (Gruyer \& coll., 1991).

Alors que les filles ont tendance à réprimer leur agressivité et à la retourner contre elles-mêmes, les garçons, quant à eux, sont plus enclins à développer des comportements violents et marginaux, et plus réticents à demander une prise en charge thérapeutique.
Les jeunes hommes ayant été abusés dans l'enfance, quant à eux, présentent davantage de risques de s'adonner à la délinquance, de faire preuve d'agressivité envers les femmes, de brutaliser voire de violer des enfants plus jeunes. Selon Rouyer et Drouet (1994), il s'agirait de la répétition des abus sur un mode actif: une tentative de passer de l'état de victime à celui d'agresseur. Selon Marie-Gimaldi (2002), il s'agirait d'un moyen d'extérioriser le traumatisme subi et impossible à verbaliser, ainsi que de se réapproprier un espace intime qui a subi l'intrusion d'un abus sexuel.

\section{La toxicomanie et l'alcoolisme.}

Outre la délinquance et l'agressivité, on retrouve également un risque accru de toxicomanie et d'alcoolisme précoce chez les adolescents victimes de violences et de carences affectives dans leur famille. Dans l'ouvrage autobiographique d'Hélène (Hélène, 2006, p.38), une adolescente en souffrance au sein d'un cadre familial négligent, il ressort que la drogue et l'alcool traduisent une volonté de combler un profond vide affectif par l'excès et la dépendance à l'objet. Rien ne vient remplir une fonction contenante pour Hélène, ni poser de limite à ses conduites à risque: "Folie, mort possible, je ne prends plus rien en compte. Je n'ai déjà plus de limites. Le danger est plus motivant que la mort elle-même. »Ce manque est pour elle impossible à mettre en sens, elle voit dans la drogue une façon de se voiler et de ne pas entendre la cause du problème (p.54): "Je sais maintenant que c'est un truc chez les toxicos. Comme la souffrance est en amont, dans l'empreinte familiale et sociale, dans cette incapacité à s'y adapter, on fait le blocus en refusant d'emblée de se retourner pour découvrir d'où elle vient.»

Dans le cas de Christiane, l'usage de la drogue vient répondre à un besoin social. C'est en effet celle-ci qui la relie à ses amis, et vient combler son désir d'affection et de reconnaissance (Felscherinow, 1981, p.73): «Ma famille c'est la bande. J'y trouve de l'amitié, de la tendresse, quelque chose qui ressemble à de l'amour. Je ne suis pas sûre qu'il existe encore une amitié comme celle qui nous lie, chez les jeunes qui ne se droguent pas.»

Ces témoignages montrent que la drogue et l'alcool chez les adolescents peuvent être un outil de destruction, 
mais aussi une échappatoire face à une société à laquelle ils ne peuvent s'adapter et à une famille qui ne peut répondre à leurs besoins affectifs. Les passages à l'acte peuvent également être un appel à l'aide qui vient exprimer une souffrance que l'adolescent ne peut pas se représenter.

\section{Dépression, troubles anxieux, phobies et conduites auto-agressives.}

L'exposition à un stress répété durant l'enfance est un facteur favorisant l'apparition de troubles anxieux chez les adolescents. II s'agit d'une angoisse irreprésentable et indicible, pouvant entraîner de nombreux symptômes somatiques tels que des troubles du sommeil ou de la digestion, cités précédemment. Des symptômes phobiques peuvent également faire leur apparition, entravant alors la vie quotidienne de façon plus ou moins importante par des comportements d'évitement et des reviviscences. Nathalie (Schweighoffer, 1990) par exemple, explique être devenue incapable de s'approcher d'une machine à laver après que son père l'ait violée sur celle de la maison familiale.

D'après l'étude de Benarous et ses collaborateurs (2014), le risque de dépression et de suicide augmente chez les adolescents ayant grandi dans un environnement familial maltraitant. Les états dépressifs, latents ou aigus, deviennent en effet une constante chez le sujet et s'expriment par un manque d'estime de soi très important entraînant des conduites autodestructrices, un manque d'attention concernant ses besoins corporels ou le traitement des maladies somatiques, voire des tentatives de suicides.

Ces comportements destructeurs peuvent constituer un signal de détresse ou bien s'avérer la seule issue à l'intolérable: mourir serait pour lui la seule façon de ne plus subir. "Moi j'attendais une mort qui ne venait pas. (...) Je la suppliais pourtant, je me mettais à genoux pour qu'elle comprenne que j'avais besoin d'elle. II fallait qu'elle me donne un coup de main, il fallait qu'elle me tue. (...)», raconte Nathalie (Scweighoffer, 1990, p.194). La pulsion de mort est en effet très ancrée dans le sujet maltraité : il s'agit de tuer son bourreau ou de se tuer soi-même. Le fantasme de meurtre, à l'instar de celui du suicide, peut alors devenir très envahissant (Schweighoffer, 1990, p.195): «Je n'avais que cette idée en tête. Tuer mon père. (...) Tout lui arracher, comme il m'a arraché ma virginité, mon corps, ma pureté, mon enfance. C'était mon rêve sadique.»

\section{Éveil de la sexualité perturbée: le corps et les affects en jeu.}

Les carences affectives en milieu familial peuvent conduire le sujet à la recherche frénétique d'attention et d'affection de la part d'autrui. Dans leur ouvrage, Rouyer et Drouet (1994) exposent le cas d'Anne, qui a reçu une éducation dépourvue de règles et de structure, couplé à des mauvais traitements quotidiens. Cet environnement maltraitant a conditionné ses rapports affectifs sur la recherche pathologique de reconnaissance et d'amour. Privée de modèles parentaux et d'interactions affectives normales, elle cherche alors à devenir un objet d'amour pour autrui à travers des rapports sexuels précoces et insatisfaisants à l'adolescence.

Si les carences affectives et les violences physiques sont dévastatrices pour le développement de la sexualité chez le sujet, les sévices sexuels le sont davantage. Premièrement, face aux transformations de son corps, l'enfant abusé qui nourrissait une image problématique de ce dernier peut ressentir une grande angoisse, voire du dégoût. Chez les filles incestées, par exemple, l'entrée dans la féminité peut être vécue comme une punition car elle ravive le traumatisme sexuel antérieur (Rouyer \& Drouet, 1994).

La notion de répression se retrouve souvent chez les adolescents victimes d'abus, par le refoulement de leurs pulsions sexuelles. Les premiers émois sont en effet souillés par les expériences précoces traumatisantes et peuvent entraîner une forte culpabilité. Cette répression des affects est souvent couplée à une conformité aux attentes de l'autre lors de l'acte sexuel: le sujet perçoit son corps comme un objet de jouissance au service du plaisir d'autrui, il ne prend donc pas en compte son propre désir. Ce mécanisme est lié au sentiment de n'avoir aucune valeur et de devoir gagner l'affection de l'autre par des faveurs sexuelles (Gruyer \& coll., 1991).

Les abus sexuels peuvent également avoir pour conséquence la pratique d'une sexualité à risque chez l'adolescent. Celle-ci peut se traduire par la multiplication des partenaires et des prises de risque 
liées aux infections sexuellement transmissibles ainsi qu'aux grossesses non désirées, des pratiques sexuelles violentes ou encore la prostitution juvénile (Gruyer \& coll., 1991; Benarous \& coll., 2014).

\section{Une prise de conscience et un dévoilement?}

Les troubles cités précédemment sont souvent interprétés par l'entourage de l'adolescent comme des difficultés liées à la période qu'il traverse, alors qu'ils révèlent en fait quelque chose de bien plus grave. L'adolescence, qui marque un désir d'affranchissement et de différentiation vis-à-vis du modèle parental, peut permettre au sujet de s'éloigner relativement de son agresseur. En voyant son emprise sur sa progéniture diminuer, celui-ci peut alors cesser ses violences physiques pour ne laisser place qu'aux violences psychologiques (Greco, 2015).

Le dévoilement des violences intrafamiliales est un processus relevant d'un cheminement individuel, plus ou moins long, selon les sujets, et il arrive qu'il ait lieu à l'adolescence.

Comme nous l'avons expliqué, dénoncer spontanément les actes commis par un parent est très compliqué pour le jeune sujet, car il ne réalise pas que ce qu'il subit est anormal, ou intériorise la culpabilité des actes de son agresseur. La prise de conscience des violences subies, voire la remémoration des scènes traumatisantes, peut être dévastatrice, car cela entraîne la levée du voile; du doute sur la réalité qui permettait au sujet de se protéger de la folie. L'équilibre précaire que constituait celui-ci est alors rompu. L'exposition soudaine de l'individu à la réalité inacceptable peut déclencher un effondrement, se traduisant par des symptômes psychotiques, des névroses ou des dépressions (Greco, 2015). Le devenir du sujet dépend alors fortement du contexte dans lequel sa révélation surviendra.

Si la dénonciation d'abus sexuels est très difficile à mettre en œuvre par la victime, elle l'est d'autant plus lorsque ceux-ci se sont produits dans le cercle de la famille (Goodman, 2003; De Voe \& Faller, 1999). Les enjeux sont en effet très importants pour la victime: celle-ci peut se taire par peur de briser la famille, opérant alors une forme de sacrifice au bon fonctionnement de cette-dernière.
Lors de violences physiques ou sexuelles perpétrés par l'un des deux parents, le comportement du conjoint lors de la découverte des sévices est décisif pour la reconstruction de la victime. Celui-ci peut manifester empathie et protection envers sa progéniture, mais aussi déni, rejet voire complicité des actes de son conjoint, ce qui opérera un double traumatisme pour la victime. La position du parent spectateur de sévices infligés à son enfant peut être ambivalente car il est possible qu'il soit partagé entre la crainte de dénoncer son conjoint et le désir de protéger son enfant, ou encore se trouver dans l'incapacité d'admettre la réalité ou subir la menace du parent maltraitant (Dupont \& coll., 2014). Toujours est-il que, si le dévoilement des violences a lieu lors de l'adolescence, le contexte familial de l'individu et le comportement de son entourage est déterminant pour sa future vie d'adulte.

\section{Les conséquences chez l'adulte}

Cette section a pour but d'expliquer la façon dont les symptômes chroniques dus à la maltraitance infantile évoluent à l'âge adulte. Tout d'abord, il est nécessaire de définir le concept de résilience : celle-ci consiste en la capacité d'un individu à maintenir une adaptation optimale malgré l'expérience d'événements déstabilisants et de conditions de vie difficiles. Cette notion peut être associée à celle du coping, qui se traduit par les stratégies mises en place par un individu pour faire face à un problème (Dardier, 2018).

Ce processus ne dépend pas seulement du milieu familial dans lequel le sujet évolue, mais aussi de son environnement scolaire, social, des relations affectives qu'il nouera au cours de sa vie, ainsi que de ses propres ressources. L'individu est donc actif dans son développement, et ne se contente pas de subir les traumatismes passés. Toutefois, l'impact des violences sur le sujet est indéniable, et celles-ci peuvent rester profondément ancrées dans sa construction psychique. Les enfants ayant été maltraités, en effet, sont reconnus comme étant plus à risque d'être victimes d'autres événements de vie difficile et d'agressions de la part d'autrui, leur fréquence étant corrélée avec la sévérité des maltraitances subies (Benarous \& coll., 2014). En plus de cela, l'impact des sévices infantiles se retrouve chez l'adulte dans de nombreux aspects de sa vie. 


\section{Les impacts sur la personnalité}

\section{Les troubles de la personnalité et les psychopathologies.}

L'étude de Johnson et ses collaborateurs (2001) démontre clairement les risques de survenue de troubles de la personnalité chez les enfants exposés à la violence, notamment à la maltraitance verbale. II peut s'agir de personnalités bordelines, évitantes, narcissiques, antisociales, dépendantes, obsessionnelles ou encore paranoïaques. On retrouve également un risque accru de développer des psychopathologies telles que la schizophrénie ou le trouble schizoaffectif. La maltraitance infantile nuit au développement cérébral, en plus d'accroître le risque de maladies somatiques ou d'autres troubles physiologiques (Anda \& coll., 2006; Chen \& coll., 2010).

L'impact de ces troubles sur la vie sociale des sujets est très lourd, car ils nuisent au bon fonctionnement des interactions et des liens affectifs. Cette faille peut représenter une entrave dans la vie amoureuse de l'adulte, mais également sur le plan professionnel en raison de résultats scolaires plus faibles dans l'enfance, d'un niveau de scolarité généralement plus bas par la suite, de plus grandes difficultés à accéder à l'emploi et des salaires moins élevés (Benarous \& coll., 2014).

\section{Perversions: I'exemple du sadomasochisme et de la perversion narcissique.}

La personnalité d'un enfant abusé se structure en réponse aux agressions sexuelles prolongées. Si l'enfant a pu ressentir du plaisir lors des abus sexuels, la forte culpabilité qui en découle donne alors lieu à un profond refoulement des pulsions et des affects, pouvant déboucher, à l'âge adulte, sur un conditionnement de la vie sexuelle sous le mode de la contrainte et de la passivité, et ainsi sur de possibles perversions (Gruyer \& coll., 1991).

Prenons l'exemple du sadomasochisme, qui est une pratique sexuelle utilisant la douleur, la domination ou I'humiliation dans la recherche du plaisir. Les mécanismes qui sous-tendent ces pulsions ne sont pas sans rappeler ceux des violences infantiles: Rubin (1999) explique, en effet, que la notion de punition et de culpabilité sont très présentes chez le sujet sadomasochiste, tout comme celle du clivage. En effet, la pulsion sadique prend source dans le clivage des mauvaises sensations du sujet, qui les projette alors de façon violente sur l'objet sexuel. La jouissance due au fait de souffrir ou de faire souffrir l'objet pourrait donc être liée aux violences subies dans l'enfance.

La perversion narcissique, quant à elle, consiste en une survalorisation de soi dans la destruction psychique d'autrui. Dans son ouvrage, Eiguer (2017) prend le cas des pères pervers narcissiques, qui considèrent l'exercice de leur autorité parentale comme un simple prestige. Pour eux, l'emprise sur les membres de la famille passe par la domination, le contrôle et la rigueur imposés via des règles et des normes qu'il s'acharne à faire respecter au quotidien. Toute désobéissance à celles-ci est source de sévères punitions. Nous retrouvons ici le principe des dictatures familiales, précédemment décrites. "Nous étions un petit peuple sous l'influence d'un tyran, d'un tortionnaire", raconte Nathalie au sujet de sa famille (Schweighoffer, 1990, p.221).

\section{La psychopathie: marginalisation et passages à l'acte.}

Les individus présentant une personnalité psychopathe ont, également, souvent été marqués par des carences affectives et des abandons de la part de leurs parents. Ils connaissent alors une marginalisation progressive et font l'objet de rejets de la part de la société. Ils éprouvent également des difficultés relationnelles avec leurs enfants, qui peuvent favoriser des passages à l'acte violents sur ceux-ci. La progéniture du sujet est, en effet, vue comme un danger, une menace pour son équilibre psychologique (Rouyer \& Drouet, 1994). L'absence de remords, de la part du parent psychopathe, pourrait donc aggraver la relation $d^{\prime}$ emprise qu'il entretient avec son enfant.

\section{La survivance du trouble de stress post-traumatique et des symptômes chroniques}

Selon Benarous et ses collaborateurs (2014), les troubles liés au stress post-traumatique perdurent à l'âge adulte et le risque de les voir subsister durant la vie entière est élevé. On peut effectivement retrouver, chez l'adulte, les mêmes symptômes que chez l'enfant maltraité et l'adolescent en souffrance (anxiété, dépression, troubles alimentaires...). Les traumatismes juvéniles sont déterminants pour la vie adulte du sujet 
et peuvent perdurer, même durant des décennies, après les événements.

Cette notion se retrouve dans le cas de Denis (Gruyer \& coll., 1991), resté bloqué psychiquement à l'âge de douze ans, date à laquelle son oncle a abusé de lui pour la première fois. Ce blocage psychique est dû au fait que son oncle n'a pas été condamné pour ses crimes, sa victime n'a donc jamais été reconnue en tant que telle, ce qui l'a empêchée de surmonter son traumatisme. Si le sujet ne bénéficie pas d'une prise en charge adaptée, le TSPT reste bien présent dans le psychisme et continue d'agir de manière latente, indépendamment du passage du temps.

\section{Les impacts sur le corps}

\section{La mémoire du corps.}

La mémoire corporelle du traumatisme est liée à la notion de répétition de l'événement et à la reviviscence des affects (Condamin, 2009). Il est possible, en effet, que le corps garde en lui les traces des violences physiques et sexuelles: ce peut être, par exemple, des douleurs chroniques aux endroits où les sévices ont eu lieu. Ferragut (2006), par exemple, présente le cas de Laura, qui souffre de douleurs chroniques dans les côtes, exactement aux endroits où son père la frappait étant petite. Ce cas illustre la façon dont le traumatisme refoulé ou inaccessible à la parole vient se manifester dans le corps. Même si le sujet a bénéficié d'un travail thérapeutique, ces douleurs peuvent subsister tels des vestiges des sévices passés.

\section{La question de la féminité dans le trauma- tisme sexuel.}

Dans leur ouvrage, Rouyet et Drouet (1994) expliquent qu'il est plus difficile de parler des conséquences des violences juvéniles sur les hommes que sur les femmes, car ceux-ci ont davantage tendance à refuser une prise en charge thérapeutique. C'est donc sur la sexualité des femmes que nous avons le plus d'éléments, et ici, en particulier sur les femmes ayant été victimes d'inceste. Toutefois, à cause du manque de données sur les sujets masculins victimes d'inceste, il est difficile de généraliser ces propos à l'ensemble des victimes d'abus.
La féminité, qui se traduit selon Perrève (2016) par les transformations physiques et psychiques qui s'opèrent chez une jeune fille en train de devenir femme, est gravement altérée par l'inceste. La distorsion de la relation entre les deux parents, couplée à un traumatisme sexuel, impacte en effet lourdement la féminité et la sexualité du sujet féminin et il peut être très difficile pour elle de s'accepter en tant que femme. Le cas de Nathalie (Schweighoffer, 1990, p.215) en est une illustration frappante: "Je me fuis moi-même, comme j'ai fui mon père, peut-être par peur de découvrir qui je suis. II a tué l'enfant que j'étais, il a tué la femme que je devais devenir, et moi je suis au milieu. Je vacille entre la gosse et la femme, sans jamais trouver ma place.»

Ce clivage de la féminité peut se traduire par le refuge dans une solitude douloureuse, le refus de tout contact physique ainsi qu'une négligence dans les soins du corps et l'habillage. A l'inverse, on peut observer une discordance entre une apparence hyper-féminine et des comportements d'évitement envers les hommes. Les traumatismes sexuels, en plus d'avoir un lourd impact sur leur féminité, agissent également sur la vie affective et sexuelle future.

\section{Les impacts sur la vie sexuelle et affective}

\section{Les troubles de la sexualité: entre inhibition et exacerbation.}

Selon Gruyer et ses collaborateurs (1991) et Gérard (2014), les troubles sexuels féminins liés aux abus juvéniles se divisent en trois pôles: le refus de la sexualité (inhibitions, dégoût, angoisse...), la dissociation entre désir et refus (frigidité, douleurs lors des rapports, manque de libido...) et l'hyperactivité sexuelle (recherche compulsive de partenaires, acte sexuel subi et dénué de plaisir). La sexualité est en effet perçue comme anormale pour le sujet ayant subi de l'inceste, et génère une intense culpabilité (Gérard, 2014). On peut également retrouver ces troubles chez les hommes, mais, dans le cas d'abus sexuels subis dans l'enfance, le risque de devenir soi-même agresseur sexuel, voire pédophile, est plus grand que chez les femmes (Pellerin, 2003; Rasho, 2018). 


\section{Les difficultés dans les relations de couple.}

Dans leurs relations amoureuses, beaucoup de sujets ne parviennent pas à établir un lien durable, s'investissent massivement dans des relations ancrées dans la dépendance affective, ou cumulent les aventures sans y trouver de satisfaction. Souvent, le sujet peine à faire confiance à son partenaire, de peur d'être à nouveau trahi et manipulé. II peut ressentir de la méfiance envers celui-ci, ou entretenir des sentiments ambivalents, d'amour, de haine et de dégoût. De plus, l'immaturité émotionnelle et l'hypersensibilité dont souffrent souvent les personnes abusées dans I'enfance sont fréquemment source de conflits au sein du couple, et le risque pour le sujet de se trouver à nouveau dans une relation d'emprise est accru (Gérard, 2014).

\section{La prostitution comme vengeance sur l'inceste.}

Un grand nombre de sujets victimes d'inceste s'adonnent plus tard à la prostitution (Gruyer \& coll., 1991; Rasho, 2018). Ces auteurs expliquent que choisir de se prostituer serait une forme de vengeance contre les agressions sexuelles subies et une vengeance contre la vie. On retrouve, dans la prostitution, la notion de monétisation de son corps présente dans l'inceste, ce qui peut avoir pour conséquences une incapacité à gagner sa vie et une absence de toute notion de l'argent. En plus de cela, on retrouve également la prise de risque liée aux infections sexuellement transmissibles, déjà évoquée chez l'adolescent.

En plus d'avoir un impact négatif sur la vie sexuelle et affective des sujets, les sévices infantiles ont également des conséquences sur la parentalité.

\section{Devenir parent lorsqu'on a subi des maltraitances infantiles}

\section{La maternité.}

Dans leur étude, Chabert et Chauvin (2005) révèlent que la maternité est à l'origine d'une réactivation de toute la pathologie traumatique ayant des conséquences sur le maternage. Ce dernier, tout comme la grossesse, risque en effet de réveiller les souvenirs traumatiques liés à l'inceste. De cela peut découler un déni de grossesse, qui agit en tant que mécanisme de défense, un abandon du nouveau-né, des maltraitances voire un infanticide.

\section{L'éducation des enfants: laxisme, abus d'autorité et répétition des violences.}

Le sujet ayant subi des maltraitances infantiles présente plus de risque de réitérer celles-ci au sein de son propre foyer (Benarous \& coll., 2014). Cette répétition provient d'un processus d'identification à l'agresseur: il s'agit là d'un mécanisme primaire mis en place par le psychisme afin de survivre aux violences subies. Ces réitérations des violences sur sa progéniture se nomment «répétitions transgénérationnelles»: c'est ainsi que les maltraitances infantiles se propagent et perdurent. Gruyer et coll. (1991) parlent de certaines familles à transactions incestueuses où les abus sont perpétrés de génération en génération: les enfants sont violés à tour de rôle, et les plus jeunes savent pertinemment qu'ils seront les prochains. Dans des cas des dictatures familiales, le risque de voir des sujets humiliés et battus tout au long de leur enfance, et de devenir à leur tour des persécuteurs pour leur famille à l'âge adulte, est non négligeable.

Le risque de répétition transgénérationnelle diffère selon les individus: tout le monde ne répète pas fatalement ses situations de victimisation, ni ne devient agresseur s'il a été exposé à la violence domestique. De nombreux facteurs entrent en jeu et influenceront le comportement futur de l'individu.

Cependant, si certains éduquent leurs enfants en répétant les abus d'autorité de leurs propres parents, d'autres tentent de les pallier en optant pour une éducation laxiste, par opposition à celles qu'ils ont reçue. Dans l'ouvrage de Christiane Felscherinow (1981), la parole est également donnée à sa mère, qui explique sa position face à la souffrance de sa fille. Elle raconte s'être montrée très permissive envers Christiane, car elle ne voulait pas la priver de ce que son propre père, alcoolique et violent, lui avait interdit dans sa jeunesse.

Avoir subi des maltraitances infantiles dans le passé est donc un facteur pouvant compliquer l'exercice de la parentalité et l'accomplissement d'une éducation saine et optimale pour ses enfants. II ne s'agit pourtant pas ici d'affirmer qu'il est impossible d'y parvenir correctement, ce processus dépendant grandement des capacités de résilience du sujet. 


\section{La résilience}

Tout le monde ne réagit pas de la même manière face à un traumatisme, et la capacité de résilience varie beaucoup d'un individu à l'autre. D'après Stephen et Joubert (2000), parmi les facteurs favorisant la résilience on retrouve l'estime de soi, la capacité d'adaptation, la créativité, I'humour, la spiritualité et les habiletés sociales. Cependant, ce processus dépend également du soutien de l'entourage social et de l'âge auquel le traumatisme survient. Concernant les maltraitances infantiles domestiques, il s'agit d'une accumulation de traumatismes, l'enfant s'est donc développé en réponse à la violence quotidienne.

La première étape du travail de résilience est, pour le sujet maltraité, de prendre conscience de son traumatisme et de reconnaître ce qu'il a subi. Pour cela, le fait de mettre des mots sur son traumatisme est primordial, d'où l'importance d'une prise en charge thérapeutique. Accepter le fait d'être victime est une chose, mais être reconnue comme telle par l'entourage, par les tribunaux et même par son agresseur lui-même peut s'avérer encore plus complexe. Pourtant, être reconnu en tant que victime est essentiel pour la reconstruction du sujet (Rasho, 2018). La condamnation du parent maltraitant est un facteur très important pouvant favoriser ce processus, mais ces violences sont rarement dénoncées, et encore moins punies (Greco, 2015).

Concernant la survivance du traumatisme, il faut préciser que chaque sujet vit à sa manière avec ce dernier, et trouve un moyen plus ou moins efficace de le surmonter. Certains se murent dans le déni, d'autres se battent pour briser le silence. Pour Nathalie, c'est le fait d'avoir fait condamner son père et d'avoir mis son vécu par écrit qui l'a aidée dans son cheminement personnel (Schweighoffer, 1990, p.241): «Depuis le temps que je jette tout sur un cahier, que j'écris comme on se lave, comme on respire l'air frais. Ce défouloir, je l'ai. Merci, en grandes lettres. Merci d'avoir fait silence en m'écoutant crier. »

\section{Conclusion}

L'objectif de cette recension était de brosser un portrait des maltraitances infantiles intrafamiliales, afin de sensibiliser sur leurs conséquences dévastatrices. II s'agissait d'expliquer le fonctionnement des violences faites aux enfants dans le cadre de la famille, et de relater leur impact sur le développement psychologique du sujet tout au long de sa vie. Les différents types de maltraitances, pouvant être le fruit de dysfonctionnements familiaux, ont été abordés, ainsi que la manière dont l'enfant se construit malgré ces sévices. L'expression des symptômes du traumatisme infantile, à travers des comportements à risque et des troubles psychologiques à l'adolescence, a ensuite été détaillée. Chez l'adulte, enfin, l'impact de la maltraitance sur les différentes sphères de la vie du sujet ainsi que sur l'exercice de la parentalité a été relaté. La survivance du trouble de stress post-traumatique et des symptômes chroniques a également été abordée. Pour terminer, les différents facteurs de résilience et les stratégies mises en place par le sujet pour faire face à son traumatisme ont été abordés.

En conclusion, les écrits présents dans la littérature scientifique permettent de comprendre les diverses conséquences de la maltraitance infantile, mais de futures recherches pourraient être effectuées afin d'approfondir certains points. II serait pertinent, par exemple, de mener davantage d'études sur l'impact de l'inceste sur le sujet masculin. Toutefois, les diverses investigations qui ont été menées sur les sévices infantiles intrafamiliaux nous permettent de comprendre en quoi celles-ci constituent un problème de santé publique alarmant, en raison des conséquences qu'elles entraînent tout au long de la vie des victimes.

\section{Références}

Anda, R. F., Felitti, V. J., Bremner, J. D., Walker, J. D., Whitfield, C., Perry, B. D., Dube, Sh. R., \& Giles, W. $H$. (2006). The enduring effects of abuse and related adverse experiences in childhood: A convergence of evidence from neurobiology, and epidemiology. European Archives of Psychiatry and Clinical Neuroscience. 256(3), 174-186. https://doi. org/10.1007/s00406-005-0624-4

Becker, E., \& Maertens, M-A. (2015). Le devenir de l'enfant victime de maltraitance sexuelle. Annales médico-psychologiques, revue psychiatrique, 9(173), 805-814. https://doi.org/10.1016/j. amp.2015.08.004 
Benarous, X., Consoli, A., Raffin, M., \& Cohen, D. (2014). Abus, maltraitance et négligence: (1) épidémiologie et retentissements psychiques, somatiques et sociaux. Neuropsychiatrie de l'enfance et de l'adolescence, 5(62), 299-312. https://doi.org/10.1016/j. neurenf.2014.04.005

Bokanowsky, T. (2002). Traumatisme, traumatique, trauma. Revue française de psychanalyse, 3(66), 745-757. https://doi.org/10.3917/rfp.663.0745

Chabert, D., \& Chauvin, A. (2005). Devenir mère après avoir été abusée sexuellement dans l'enfance. Neuropsychiatrie de l'enfance et de l'adolescence, 1-2(53), 62-70. https://doi.org/10.1016/j. neurenf.2004.09.009

Chen, L. P., Murad, M. H., Paras, M. L., Colbenson, K. M., Sattler, A. L., Goranson, E. N., Elamin, B.E., Seime R.J., Shinozaki, G., Prokop, L.J. \& Zirakzadeh, A. (2010). Sexual abuse and lifetime diagnosis of psychiatric disorders: Systematic review and meta-analysis. Mayo Clinic Proceedings, 7(85), 618-629. https:// doi.org/10.4065/mcp.2009.0583

Cherifi, Z. (2008). Du traumatisme au lien psychique. Pratiques psychologiques, 4(14), 471-480. https:// doi.org/10.1016/j.prps.2008.02.001

Chiland, C., Noizet, Y., Young, G., \& Kaplan, D. (1997). Les enfants et la violence. Paris: Presses universitaires de France.

Chraïbi, S. (2006). Thérapeutique du syndrome de répétition chez l'enfant et l'adolescent victimes d'agressions sexuelles. Annales médicopsychologiques, 2(164), 134-140. https://doi. org/10.1016/j.amp.2005.12.004

Ciccone, A., Gauthier, Y., Glose, B., \& Stern, D. (2008). Naissance et développement de la vie psychique. Toulouse: Ères.

Cohn, J.F., \& Tronick, E. (1989). Specificity of infants' response to mothers' affective behavior. Child Adolescent Psychiatry, 2(28), 242-248. https://doi. org/10.1097/00004583-198903000-00016

Condamin, C. (2009). Trauma, résilience et mémoire du corps. Bulletin de psychologie, 5(503), 457-466. https://doi.org/10.3917/bupsy.503.0457

Crocq, L. (2014). Traumatismes psychiques, prise en charge psychologique des victimes. Issy-les-Moulineaux: Elsevier Masson.
Hélène, H. (2006). J'ai commencé par un joint. Paris: $\mathrm{OH}$ ! Editions.

Dardier, V. (2018). Psychologie du développement. Recueil inédit, Rennes 2.

Définition de l'inceste. (2012). Consulté à l'adresse https://www.cnrtl.fr/

Delion, P., (2011). La fonction parentale. Paris: Fabert.

DeVoe, E-R., \& Faller, K-C. (1999). The characteristics of disclosure among children who may have been sexually abused. Child Maltreat, 4(3), 217-227. https://doi.org/10.1177/1077559599004003003

Dugravier, R., \& Barbey-Mintz, A-S. (2015). Origine et concepts de la théorie de l'attachement. Enfances \& Psy, 2(68), 14-22. https://doi.org/10.3917/ ep.066.0014

Dupont, M., Messerschmitt, P., Vila, G., Bohu, D., \& Rey-Salmon, C. (2014). Le processus de révélation dans les agressions sexuelles intrafamiliales et extrafamiliales sur mineurs. Annales médicopsychologiques, 6(172), 426-431. https://doi. org/10.1016/j.amp.2012.06.024

Eiguer, A. (2017). Les pervers narcissiques. Paris: Presses Universitaires de France.

Felscherinow, C. (1981). Moi, Christiane F., 13 ans, droguée, prostituée... Paris: Folio.

Ferragut, E. (2006). Agressions \& maltraitance. Issy-les-Moulineaux: Elsevier Masson.

Gérard, C. (2014). Conséquences d'un abus sexuel vécu dans l'enfance sur la vie conjugale à l'âge adulte. Carnet de notes sur les maltraitances infantiles, 1(3), 42-48. https://doi.org/10.3917/cnmi.132.0042

Greco, C. (2015). Maltraitance faite aux enfants: entre méconnaissance du problème et déni. Ethics, medecine and public health, 1(1), 11-18. https://doi. org/10.1016/j.jemep.2015.01.001

Gruyer, F., Sabourin, P., \& Fadier-Nisse, M. (1991). La violence impensable: inceste et maltraitance. Paris: Nathan.

Guillet, L. (2012)., Le stress. Paris: Boeck Supérieur.

Jan, O. (2001) Traumatisme infantile et actuel. Vie sociale et traitements, 2(70), 29-32. https://doi. org/10.3917/vst.070.0029 
Johnson, J.G., Cohen, P., Smailes, E.M., Skodol, A.E., Brown, J., \& Oldham, J.M. (2001). Childhood verbal abuse and risk for personality disorders during adolescence and early adulthood. Comprehensive Psychiatry, 1(42), 16-23. https://doi.org/10.1053/ comp.2001.19755

Kim Oates, R., Forrest, D., \& Peacock, A. (1985). Self-esteem of abused child. Child abuse and Neglect, 2(9),159-163. https://doi.org/10.1016/0145-2134(85)90007-9

Krug, E., Dahlberg, L., Mercy, J., Ziwi, A., \& LozanoAscencio, R. (2002). Rapport mondial sur la violence et la santé. OMS. Récupéré sur https://apps.who.int/ iris/bitstream/handle/10665/42495/9241545615_ eng.pdf;sequence $=1$

Le Breton, D. (2007). En souffrance: adolescence et entrée dans la vie. Paris: Editions Métailié.

Le trouble de stress post-traumatique (TSTP). (2019). Consulté à l'adresse www.institutdevictimologie. fr/trouble-psychotraumatique/maltraitance-infantile_29.html

Lebigot, F. (2016). Traiter les traumatismes psychiques. Malakoff: Dunod.

Lévi-Strauss, C. (1986). Histoire de la famille. Paris: A. Colin.

Lévy, I. (2008). Le jeune enfant face à sa culture ou sa religion. La lettre de l'enfance et de l'adolescence, 4(74), 73-78. https://doi.org/10.3917/lett.074.0073

Marcelli, D. (2014). La «trace anti-mnésique», hypothèse sur le traumatisme psychique de l'enfant. L'information psychiatrique, 6(90), 439-446. https://doi. org/10.3917/inpsy.9006.0439

Mariani, A., Chalies, S., Jeandel, C., \& Rodière, M. (2010). Le nanisme psychosocial, une réalité toujours d'actualité. Archives de pédiatrie, 5(17), 486-490. https://doi.org/10.1016/j.arcped.2010.02.006

Marie-Grimaldi, H. (2002). Abus sexuels et comportements violents chez des enfants placés à l'aide sociale à l'enfance. Neuropsychiatrie de l'enfance et de I'adolescence, 3(150), 193-200. https://doi. org/10.1016/S0222-9617(02)00091-0

Melgual, C. (2019). Adolescence, corps et violence. Recueil inédit, Rennes 2.

Montandon, C., \& Kellerhals J. (1991). Les stratégies éducatives des familles. Paris: Delachaux et Niestlé.
Parat, H. (2011). Les destins de l'inceste. L'évolution psychiatrique, 2(76), 245-261. https://doi. org/10.1016/j.evopsy.2011.03.016

Pellerin, B. (2003). La théorie de l'abusé-abuseur en délinquance sexuelle: qui dit vrai? Canadian Journal of Criminology and Criminal Justice, 1(45), 81. https://doi.org/10.3138/cjccj.45.1.81

Perrève, C. (2016). Au seuil de la féminité. Revue de l'enfance et de l'adolescence, 2(94), 179-192. https://doi.org/10.3917/read.094.0179

Rasho, A-B. (2019). Victimologie. Recueil inédit, Rennes 2.

Razo, L., \& Metz, C. (2011). La violence et son devenir chez l'enfant témoin de violences conjugale. Neuropsychiatrie de l'enfance et de l'adolescence. 7(59), 411-414. https://doi.org/10.1016/j. neurenf.2011.08.005

Rosenbulm, O. (2000). Les enfants et leurs parents toxicomanes. Journal de pédiatrie et de puériculture, 7(13), 405-411. https://doi.org/10.1016/S09877983(00)80035-3

Rouyer, M., \& Drouet, M. (1994). L'enfant violenté: des mauvais traitements à l'inceste. Paris: Bayard.

Rubin, G. (1999). Le sadomasochisme ordinaire. Paris: L'Harmattan.

Scharbach, H. (2018). Syndrome de stress post-traumatique et B-Bloquants (2018). Annales médico-psychologiques, revue psychiatrique, 5(176), 523-525. https://doi. org/10.1016/j.amp.2018.03.007

Schweighoffer, N \& Cuny M-T. (1990). J'avais douze ans... Paris: Fixot.

Solis-Ponton, L. (2002). La parentalité, défi pour le troisième millénaire. Paris: Presses Universitaires de France.

Thomas-Antérion, C. (2012). L'amnésie dissociative: une rare situation de voyage dans le temps perdu. Annales médico-psychologiques, revue psychiatrique, 3(170), 181-184. https://doi.org/10.1016/j. amp.2012.02.004

Vuaillat, S., \& Ferenczi, S. (2009). Entre réalité psychique et réalité du traumatisme: pertinences et butées de son œuvre dans la clinique. Perspectives psy. 4(48) 363-374. Récupéré sur https://www.cairn.info/ revue-perspectives-psy-2009-4-page-363.htm 
Widmer, E., Kellerhals, J., \& Lévy R. (2004). Quelle pluralisation des relations familiales? Revue française de sociologie, 1(45) 37-67. https://doi.org/10.3917/ rfs. 451

\section{Pour citer l'article}

Wattel, I. (2020). Portrait des conséquences associées aux maltraitances infantiles intrafamiliales à partir d'une recension d'écrits scientifiques. Psycause: Revue scientifique étudiante de l'École de psychologie de l'Université Laval, 10(1), 28-44.

\section{Droits d'auteur}

(C) 2020 Wattel. Cet article est distribué en libre accès selon les termes d'une licence Creative Commons Attribution 4.0 International (de type CC-BY 4.0) qui permet l'utilisation du contenu des articles publiés de façon libre, tant que chaque auteur ou autrice du document original à la publication de l'article soit cité(e) et référencé(e) de façon appropriée. 